\title{
Time Lags of Horizontal Branch Oscillations in Z Source GX 5-1
}

\author{
Jin-Lu Qu ${ }^{1}$, Guo-Qiang Ding ${ }^{1}$, Jian-Feng $\mathrm{Ji}^{2}$, and Ming-Xuan Zhang ${ }^{1}$ \\ ${ }^{1}$ Laboratory for High Energy Astrophysics, Institute of High Energy \\ Physics, CAS, Beijing 100039, P. R. China \\ ${ }^{2}$ Depart of Engineering Physics, Tsinghua University, Beijing 100084, \\ P. R. China
}

\begin{abstract}
We investigated the time lags and the evolution of the cross spectrua of Z source GX 5-1, observed by Rossi X-ray Timing Explorer (RXTE), when it is in the horizontal branch oscillations. We showed that the time lags of 3 horizontal branch oscillations are related to the position on the hardness intensity diagram. 3 QPOs were shown to be of the hard time lags. But on the cross spectra, one is in a "dip", one in a "bump", another has not that chracteristic. The time lags of two of the QPOs decrease with QPO's frequency, and one has a trend increasing with its freuqency. In the normal branch, we found that there is no significant time lags in the present observational data.
\end{abstract}

\section{Introduction}

The neutron-star low-mass X-ray binaries (LMXBs) can be divided into two types on the basis of pattern traced out in the X-ray intensity-hardness or colorcolor diagram (CCD or HID, respectively), one is $\mathrm{Z}$ sources which traced out a "Z" pattern on HID or CCD, another is atoll sources which traced out a "C" pattern on CCD. From top to bottom of the Z pattern for a $\mathrm{Z}$ source, the three limbs of the pattern traced out on HID or CCD is called Horizontal Branch (HB), Normal Branch (NB), and Flaring Branch (FB). The temporal properties of $\mathrm{Z}$ sources are associate with the position on the $\mathrm{Z}$ pattern.

Using EXOSAT and Ginga observational data, Van Der Klis et al. (1987) and Vaughan et al. $(1994,1999)$ had studied the time lags (or phase lags) of two QPO components on the HB and low frequency noise (LFN). Using RXTE observational data,Jonker et al. (2002) studied in detail the powerdensity spectra (PDSs) of the Z source GX 5-1. They showed that there are 4 QPOs on the HB, HBO and its harmonics.

Because the launch of the Rossi X-ray Timing Explorer (RXTE) with large effective collective area and high time-resolution, we can investigate the crossspectrum and PDSs of GX 5-1 in more detail. In this paper, we reported the time lag of HBO and its harmonics by RXTE observational data. 


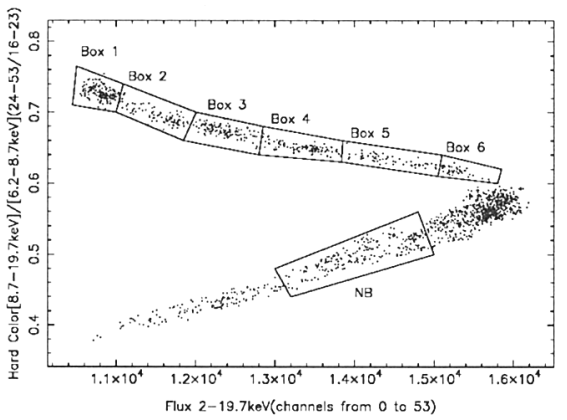

Figure 1. The HID of GX 5-1. Each point corresponds to $32 \mathrm{~s}$ of data from 5 PCUs.
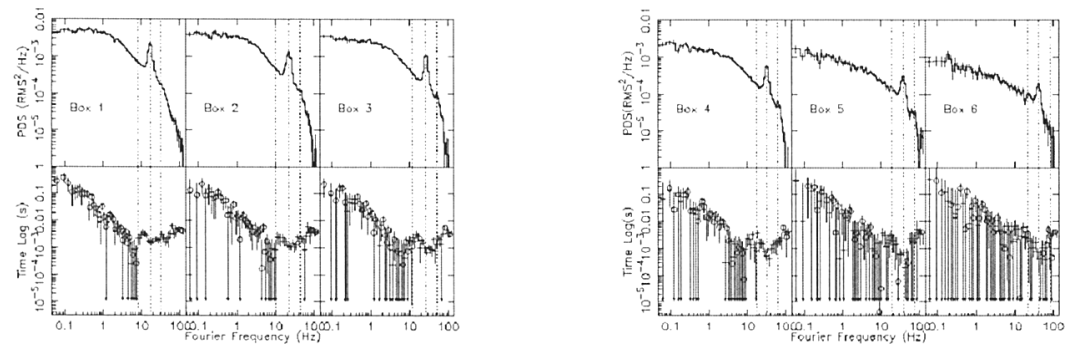

Figure 2. The PDSs for $8.7-60 \mathrm{keV}(t o p)$ and cross spectra between $2-5.1 \mathrm{keV}$ and $8.7-60 \mathrm{keV}$ (bottom) of GX 5-1.

\section{Observations and Analysis}

GX 5-1 was observed 11 times in 1997 with the Proportional Counter Array(PCA) on board the RXTE satellite. Because of different energy channel bins in different observation epoch, we only used 6 times of observational data from May 30 to July 25, 1997.

The standard 2 data was used to make a HID. The hardness, or hard color, and intensity were defined as the $8.7-19.7 \mathrm{keV} / 5.9-8.7 \mathrm{keV}$ count rate ratio and as the count rate in the $2-19.7 \mathrm{keV}$ band of a $32 \mathrm{~s}$ in average, respectively. The HID was showed in Fig.1, each point represents $32 \mathrm{~s}$ data length in the HID. The source was found on the HB, and the NB. To investigate X-ray temporal properties of GX 5-1 along the Z track, we divided the HB into 6 boxes (see Fig.1).

Using high time-resolution data, we calculated the power-density spectra for each energy band and cross-spectra for two energy bands of data stretches of $32 \mathrm{~s}$ with $4 \mathrm{~ms}$ time bin in each box, and the dead-time effect was corrected (van der Klis et al 1987; Qu, Yu, \& Li 2001). The PDSs and the cross spectra are shown in Fig.2 


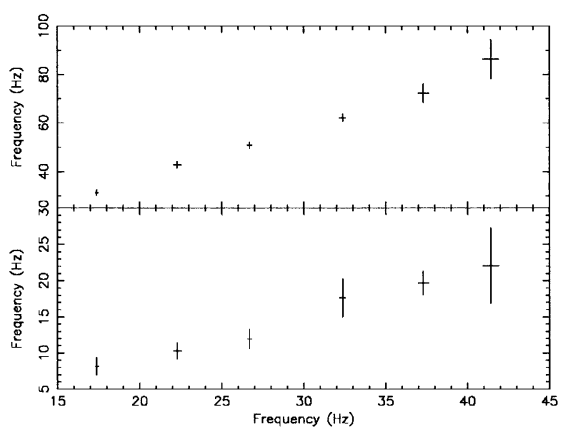

Figure 3. The relations among HBO and sub-HBO (bottom)) as well as the harmonic $(t o p)$.

\section{Results and Discussions}

Because of less data than Jonker's (2002) and low signal-to-noise in high frequencies, we only used a model composed of three Lorentzians (i.e., 3 QPO components) and a cut-off power-law to fit the PDSs of GX 5-1. Following Jonker's, we called the low-frequency QPO as sub-HBO, the strongest QPO as $\mathrm{HBO}$, the higher frequency QPO as the harmonic. In Fig.2, QPOs' position (QPO's centroid frequency) were indicated by the dotted-lines. As illustration, we plotted the $\mathrm{HBO}$ vs. sub-HBO and harmonic in Fig.3.

The cross spectra (Fig.2; panel 1-6, bottom) showed that the time lags of the noise less than $10 \mathrm{~Hz}$ were dominated by soft time lags (negative lags). During QPOs' frequency range, GX 5-1 shows hard time lags (positive lags). Those results were consistent with early works(van der Kils et al. 1987; Vaughan et al. 1994). But the time lags of QPOs show the different and more complex timing behaviors. For example, HBO's time lags may be in a "dip" and harmonic in a "bump". The sub-HBO also may be different with HBO's and the harmonic's behavior, which has a trend to increase with the sub-HBO frequency.

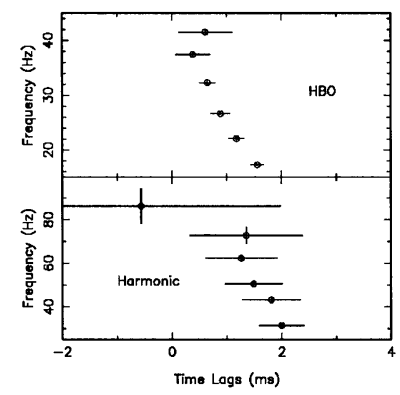

Figure 4. Time lags of HBO and the harmonic.

Fig.4 showed the time lags of the HBO and the harmonic. The time lags of the sub-HBO were plotted in Fig.5. Because the sub-HBO was in frequency range of the LFN, time lags of the LFN would affect the sub-HBO. We fitted 


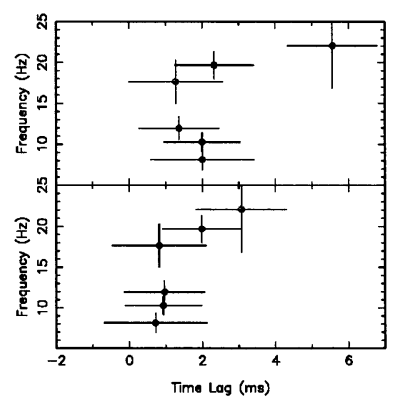

Figure 5. Time lags of sub-HBO. The top is the time lags corrected with LFN's by a power-law model.

the time lags between 0.2 and $4 \mathrm{~Hz}$ by a power-law model. The time lags of the LFN were subtracted from the sub-HBO's based on the power-law model. The uncertainties were not modified. In analyzed RXTE observational data and energy bands, we didn't find the significant time lags between NBO's frequency range.

To explain the measured time lag in the compact objects, there some kinds of models were proposed (Poutanen 2000). In the Comptonization models, e.g., the uniform corona model (Payne 1980), the non-uniform corona model (Kazanas 1997), and the drifting-blob model (Bottcher \& Liang 2001), only explain the hard time lags. Those models also have physical problems(see the review by Poutanen 2000).

To explain the measured soft time lags and time lag evolution in the sources GRS 1915+105 and Cir X-1 (Cui 2000; Reig et al. 2000; Qu, Yu \& Li 2001), a two layers of corona model has been proposed (Nobili et al. 2000, Qu Yu \& $\mathrm{Li}$ 2001). In this model, the evolution of time lags with mass accretion can be explained, but the time lag of the LFN and HBOs and NBO can not explained in GX 5-1 simultaneously.

Both shot profile properties and Comptonization of photons can introduce time lags. The simulative calculation showed that energy-dependent shot profiles can produce low-energy phase lags in the cross spectrum at frequencies typical of the shot time scale (tenths of a Hertz to a few Hertz) without noticeably afftecting the cross spectrum at higher frequencies(Shibazaki et al. 1998). Although shot models can produce almost any time-delay spectrum, but like Comptonization models, they have problems on physical grounds(Vaughan et al. 1999).

Acknowledgments. J.L. Qu thanks the J.-M. Wang for his reading the manuscript. We are grateful to $\mathrm{R}$. Staubert for help in downloaded the data. This work was subsized by the Special Funds for Major State Basic Research Projects and by the Natural Science Foundation of China.

\section{References}

Böttcher M., \& Liang E. P. 1999, ApJ, 511, L37 
Cui W. 1999, ApJ, 524, L59

Kazanas D., Hua X. -M., \& Titarchuk L. 1997, ApJ, 480, 735

Jonker P.G., van der Klis M., Homan J., et al. 2002,MNRAS, astro-ph/0202420

Nobili L., Turolla R., Zampieri L., \& Belloni T. 2000, ApJ, 538, L137

Payne D. G., 1980, ApJ, 237, 951

Poutanen J., astro-ph/0002505

Qu J.L., Yu W.,\& Li T.P. 2001, ApJ, 555, 7

Reig P., Belloni T., van der Klis M., et al. 2000, ApJ, 541, 883

Shibazaki N., Elsner R. F., Bussard R. W., et al. 1988, ApJ, 331, 247

van der Klis M., Hasinger G., Stella L., et al. 1987, ApJ, 319, L13

Vaughan M., van der Klis M., Wijers R.A.M.J., et al. 1994, ApJ, 421, 738

Vaughan B.A., van der Klis M., Lewin W. H. G., et al. 1999, A\&A, 343, 197 